Meta

Journal des traducteurs

Translators' Journal

\title{
Linguistique et traduction : propos de traducteur
}

\section{Ton That Thien}

Volume 28, numéro 2, juin 1983

URI : https://id.erudit.org/iderudit/004474ar

DOI : https://doi.org/10.7202/004474ar

Aller au sommaire du numéro

Éditeur(s)

Les Presses de l'Université de Montréal

ISSN

0026-0452 (imprimé)

1492-1421 (numérique)

Découvrir la revue

Citer cet article

Thien, T. (1983). Linguistique et traduction : propos de traducteur. Meta, 28(2), 130-152. https://doi.org/10.7202/004474ar d'utilisation que vous pouvez consulter en ligne.

https://apropos.erudit.org/fr/usagers/politique-dutilisation/ 


\section{LINGUISTIQUE ET TRADUCTION : PROPOS DE TRADUCTEUR}

TON THAT THIEN

Pendant longtemps, linguistes et traducteurs ont travaillé séparément et se sont ignorés mutuellement. C'est seulement récemment que l'idée d'une association entre linguistique et traduction a été lancée. Parmi ceux qui préconisent une telle association Georges Mounin est sans doute le plus ardent. Pour lui, l'ignorance complète de l'existence de la traduction par tous les traités de linguistique est «le plus grand scandale de la linguistique contemporaine ${ }^{1}$ "; en même temps, il invite les traducteurs à faire «une réflexion linguistique» sur la traduction $^{2}$. L'idée d'une association linguistique-traduction a fait son chemin, et, aujourd'hui, un jumelage de ces deux disciplines semble même naturel et nécessaire à certains.

Évidemment, les questions d'ordre linguistique ne sont jamais absentes des réflexions des traducteurs. On les trouve déjà chez Saint Augustin (354-430) et chez les Romains ${ }^{3}$. D'autre part, la traduction a fait une entrée remarquée en linguistique, par le biais de l'anthropologie et de la sémantique, avec l'introduction de la notion de «contexte de situation» par B. Malinowski, dans une étude publiée en supplément dans un livre de C.K. Ogden et I. A. Richards paru en $1921^{4}$. La «jonction» entre la linguistique et la traduction est réalisée en fait en 1945 par E. A. Nida, de l'American Bible Society, mais l'idée d'une association formelle entre la linguistique et la traduction n'a été émise qu'en 1949 par A. V. Feodorov, qui préconisait une intégration de la traduction à la linguistique, idée qui sera suggérée également par J.-P. Vinay et J. Darbelnet en $1958^{5}$.

L'idée de l'association entre linguistique et traduction vient surtout des linguistes. Qu'elle ait germé chez ces derniers et ait fait son chemin plus vite chez eux est chose naturelle. Les linguistes doivent, bon gré mal gré, s'occuper de théorie, et il semble admis à présent que la théorie linguistique doit faire une place à la traduction. En fait, le nombre de linguistes qui se

\footnotetext{
* Note sur les citations: Parmi les ouvrages cités en référence, il y en a qui ont été traduits en français, et d'autres non. Dans le premier cas (les titres sont donnés en français) les citations sont des traducteurs des ouvrages en question. Dans le second cas (les titres sont dans la langue originale) les traductions sont de moi-même.

1. Georges Mounin (1963) : les Problèmes théoriques de la traduction, Paris, Gallimard, p. 8.

2. Id. (1976) : Linguistique et traduction, Bruxelles, Dessart et Mardaga, p. 8.

3. Louis Kelly (1979) : The True Interpreter, New York, Saint Martin's Press, p. 1-2.

4. C.K. Ogden et I.A. Richards (1976): The Meaning of Meaning, New York, Harcourt, Brace and World Incorp., (1963). L'étude de Malinowski est intitulée: «The Problems of Meaning in Primitive Languages».

5. Mounin, Linguistique et traduction, p. 80 .
} 
sont mis à discourir sur ce sujet s'est considérablement accru récemment. Pour certains c'est peut-être une découverte excitante ; pour d'autres, c'est une trouvaille, un champ d'étude nouveau, virtuellement inexploré.

Les traducteurs, par contre, ne se passionnent pas généralement pour les questions de théorie, et encore moins, de théorie linguistique. Pour certains, c'est une question de tempérament; pour d'autres, de formation; pour la majorité, ceux qui ont choisi la traduction comme gagne-pain, une question de finalité et de temps. Ce n'est qu'avec l'essort de l'enseignement de la traduction que les questions théoriques ont pris de plus en plus d'importance, car, inévitablement, ceux qui enseignent cette matière en ont une certaine conception théorique et, tôt ou tard, par goût ou par contrainte, ils seront amenés à expliciter cette conception. C'est ainsi, par exemple, qu'est née la traductionologie au Canada ${ }^{6}$, où l'enseignement de la traduction a pris un essort considérable depuis la fin de la seconde guerre mondiale. Pour la majorité des traducteurs, cependant, le chemin de la traduction à la linguistique semble long et ardu. Certains d'entre eux se demandent s'il faut parcourir ce chemin et «faire de la linguistique». Il y a des raisons pour et contre cela.

Parmi les premières, on peut citer le sentiment de «noblesse oblige». Le traducteur, puisqu'il manie les langues, n'est-il pas «linguiste», lui aussi? Et peut-il prétendre être «linguiste» s'il ignore tout de la linguistique? Viennent ensuite les considérations pratiques, d'ordre professionnel. Les traducteurs entendent dire parfois qu'une connaissance de la linguistique est utile, ou même indispensable, au traducteur. Si cela est vrai, n'est-il pas une obligation pour le traducteur d'étudier la linguistique? Il existe une troisième raison, moins forte que les deux premières, mais importante quand même : la connaissance de la linguistique ne doit-elle pas faire partie de la culture générale d'un homme contemporain?

Contre les raisons mentionnées, il y en a d'autres qui incitent à l'hésitation. D'abord, la linguistique ne risque-t-elle pas d'être seulement une matière «à la mode», comme le dit André Martinet, lui-même linguiste ${ }^{7}$ ? Ensuite, la linguistique semble être une matière très théorique, et le traducteur, en entreprenant son étude ne risque-t-il pas de faire un piètre investissement de son énergie et de son temps? Enfin, la linguistique est apparemment un domaine très vaste, et, à supposer que le traducteur se décide à y pénétrer, par où faut-il commencer?

Les questions ci-dessus sont sans doute posées par beaucoup de traducteurs. Elles sont légitimes, et il faut y répondre. C'est ce que je vais essayer de faire. Je le ferai, et je tiens à le souligner, comme traducteur, et non comme linguiste - ce que je ne suis pas. En tant que traducteur qui s'est posé les

6. D'autres préfêrent «traductologie», mais, à mon avis, «traductionologie» est plus facilement identifiable avec «traduction». Termes qui vont avec lui : traductif (activité traductive - plutôt que «traduisante» -); traductionnel (opération traductionnelle); traductionologique (considération traductionologique); traductionologue. J'emploierai également LO et LT pour langue de l'original et langue de traduction. D'autres préferent LD et LA (langue de départ et langue d'arrivée) ou LS et LC (langue source et langue cible). À mon avis, le doublet LO/LT a l'avantage de convenir en anglais aussi bien qu'en français, avantage très appréciable au Canada.

7. André Martinet, dans Jeanne Martinet (1972): De la théorie linguistique à l'enseignement des langues, Paris, PUF, p. 57. 
mêmes questions, et qui a parcouru un bon bout du chemin de la traduction à la linguistique, je pense pouvoir offrir des réponses, au moins partielles, à ces questions. Mes propos sont ceux d'un traducteur qui s'adresse à d'autres traducteurs. D'autre part, comme le titre de cet article l'indique, je ne prétends pas parler au nom de tous les traducteurs, mais je suis quand même convaincu que beaucoup d'entre eux partageront mon point de vue, et, enfin, je suis poussé non par un «pathos théoriciste» quelconque (pour emprunter le terme de J.R. Ladmiral(8)), mais simplement par la curiosité.

Ce que le traducteur attend de la linguistique dépend des exigences qui découlent de la nature de son travail. Celui-ci consiste à réexprimer dans la LT (langue de traduction), aussi complètement que possible, le message exprimé dans la LO (langue de l'original). Le produit de ce travail est un texte dans la LT équivalent au texte dans la LO, c'est-à-dire dont la teneur est l'équivalent, ou la meilleure approximation possible, de celle de la LO. Pour bien faire son travail, le traducteur doit savoir manier parfaitement les langues dans lesquelles il a choisi d'exercer son métier. Mais, à la différence de l'interprète, qui est soumis à des contraintes différentes, la langue que le traducteur doit savoir manier à perfection est la langue écrite. Il doit comprendre parfaitement ce qu'il lit, et exprimer avec aisance ses idées par écrit. Cela veut dire qu'il doit être rompu à la reconnaissance et à l'interprétation des signes visuels, plus spécifiquement des signifiants graphiques, en particulier des marqueurs grammaticaux graphiques; autrement dit, le traducteur doit être expert en morphographologie.

Deuxièmement, le message exprimé dans la LT doit être équivalent à celui exprimé dans la LO. Cette équivalence est une équivalence fonctionnelle, rhétorique, ou, pour emprunter le terme utilisé par Nida, «dynamique ${ }^{9} »$. Pour cela, l'analyse doit s'appliquer au texte entier et pris dans son ensemble. L'objet d'analyse du traducteur n'est donc pas un syntagme, une phrase, ou même un paragraphe, mais le discours entier: c'est l'unité normale d'analyse, le paragraphe et la phrase étant, respectivement, l'unité intermédiaire et l'unité minimale. Le traducteur doit interpréter le discours en s'appuyant non seulement sur l'analyse lexicale et syntaxique, mais aussi, et surtout, sur la rhétorique, comme l'a souligné Jean Delisle dans une étude qui, sans doute, reflète le point de vue de la grande majorité des traducteurs. "La traduction, dit-il, est un exercice interprétatif, une analyse intelligente du discours ${ }^{10}$ », et c'est «la rhétorique, étant définie comme l'étude du discours, qui doit fournir le cadre théorique... à la traduction ${ }^{11} \gg$. Or, le discours fait partie du domaine de la parole (ou performance) et non de la langue (ou compétence). Le discours, enfin, déborde non seulement du cadre de la phrase, mais même du texte. Il doit être placé dans un contexte spécifique, dans le sens firthien du terme,

8. J.R. Ladmiral (1979): Traduire: Théorèmes pour la traduction, Paris, Payot, p. 9.

9. Eugene A. Nida (1964): Toward a Science of Translating, Leiden, J.B. Brill, p. 159 et ss.

10. Jean Delisle (1980): l'Analyse du discours comme méthode de traduction, Ottawa, Presses de l'Université d'Ottawa, p. 65.

11. Ibid., p. 60 . 
c'est-à-dire dans un combiné de beaucoup d'éléments : «les gens, les choses, et les événements dans le contexte de situation, l'ensemble des facteurs culturels qui conditionnent le locuteur, et tous les facteurs culturels qui forment le cadre du langage ${ }^{12} »$.

Troisièmement, la préoccupation principale du traducteur, quand il aborde un texte, est le sens. Sauf pour un genre très spécial, la poésie, le sens passe avant la forme. Entre le sens et la forme, le sens a priorité. Cette règle de la primauté du sens est connue de tous les traducteurs. Elle est absolue dans les traductions utilitaires («pragmatiques», si l'on emploie le terme de Delisle), c'est-à-dire celles dont le but principal est l'information.

Toute opération traductionnelle comporte trois phases: $l$ ) compréhension (interprétation ou décodage), 2) traduction proprement dite (ou changement de code), et 3) reformulation (mise en forme, ou encodage). Si la seconde, la traduction proprement dite, est inter-linguale, la première et la troisième sont intra-linguales (conversion en paraphrases et conversion de paraphrases); la première est «sémasiologique» (de la forme au sens), et la troisième «onomasiologique (du sens à la forme). ${ }^{13}$ Dans la première, le but visé est la compréhension totale - la saisie du sens - du texte. Dans la dernière, c'est encore le souci de préservation de la totalité du sens qui doit guider dans le choix des formes. La sémantique est donc au centre des préoccupations du traducteur.

Viennent ensuite la syntaxe et le lexique. Des deux, c'est le lexique qui constitue le souci majeur du traducteur. Les règles de syntaxe sont en nombre fini. La grammaire est un ensemble fermé. Elle peut être maitrisée au bout d'un certain temps. La durée de l'acquisition de cette maîtrise varie, il est vrai, selon les dons de chacun en matière de langue, mais elle est finie. Le lexique, c'est une tout autre affaire. Personne ne peut prétendre, ou espérer, connaitre tous les mots d'une langue, y compris sa langue maternelle, et encore moins tous les sens de tous les mots de cette langue, parce que non seulement le nombre des mots est extrêmement grand, mais aussi, et surtout, parce que les mots entrent dans des combinaisons infiniment variées. D'autre part, les mots forment un ensemble ouvert: on en crée de nouveaux constamment. Une autre difficulté, encore plus grande, est que les mots prennent des sens différents selon les contextes et, dans le domaine de la parole, ces contextes sont infiniment variés et variables.

Le problème se complique encore parce que les unités lexicales (mots ou groupes de mots) véhiculent des référents et des connotations différents selon les cultures, et que, entre unités lexicales, s'il n'y a pas de vraies correspondances de forme, il y a encore moins de vraies correspondances de sens. C'est le problème de culture: différences de vision du monde, de découpage de la réalité (au niveau collectif et individuel). Le traducteur doit être donc une

12. J.R. Firth (1968): The Tongues of Men and Speech, London, Oxford University Press, (1930/37), p. 88.

11. Les termes "sémasiologie" et "onomasiologie " sont empruntés à Charles Bouton (1979) : Linguistique appliquée, Paris PUF, "Que sais-je?", p. 61. Mais Bouton distingue seulement deux phases en traduction. 
personne non seulement vraiment bilingue, mais aussi vraiment biculturelle (pour ne parler que des traductions qui mettent en jeu seulement deux langues).

En plus du problème de culture, le traducteur doit faire face au problème de matière. C'est un fait bien connu en traduction qu'aucun traducteur ne peut être universellement polyvalent, et qu'il doit se spécialiser. Pour bien traduire, il faut connaître la matière. C'est ce que Étienne Dolet a déjà souligné avec force il y a déjà quelque cinq siècles. "Il faut, dit-il, que le traducteur entende parfaitement le sens et la matière de l'auteur ${ }^{14}$ ». Dolet estime cette règle si importante qu'il l'a placée en tête des cinq règles de la manière de bien traduire. Or connaître une matière veut dire connaître les problèmes, les concepts, et la terminologie qui vont de pair avec elle, et le traducteur doit connaître non pas une, mais plusieurs matières: ses difficultés sont multipliées d'autant.

L'acquisition de la maitrise lexicale est donc une des grandes nécessités en traduction. Elle ne peut se faire que si le traducteur se familiarise avec le maximum de contextes par la lecture de toutes sortes de textes portant sur toutes sortes de sujets. En d'autres termes, il faut que le traducteur se plonge en plein dans le domaine de la parole. C'est cette lecture qui, avec l'étude de la littérature et de l'analyse littéraire, lui permettra d'acquérir le sens de la langue qu'aucune science de la langue ne pourra lui donner. C'est elle qui lui donnera l'intuition linguistique dont il aura grandement besoin dans la phase sémasiologique comme dans la phase onomasiologique.

Ce qui précède explique pourquoi, à moins d'avoir un penchant spécial pour l'abstraction et pour la généralisation, ou à moins d'y être contraint par des nécessités pédagogiques - ce qui n'est pas le cas de la majorité - le traducteur n'aborde pas usuellement l'étude des langues sous l'angle théorique. Il est, rappelons-le, un linguiste praticien, «un technicien du langage» comme dit Delisle $^{15}$. Ce n'est donc pas vers la théorie linguistique et la théorie grammaticale mais vers la linguistique appliquée et la grammaire normative (prescriptive) qu'il se tournera, par instinct autant que par nécessité.

Face aux besoins spécifiques ci-dessus - parmi d'autres - le traducteur qui regarde vers la linguistique, en particulier la linguistique contemporaine, se demande ce que celle-ci peut lui offrir pour l'inciter à investir une partie de son temps et de son énergie dans l'étude d'un domaine aussi vaste qu'étrange, à s'engager dans une immense forêt où les risques de se perdre sont grands. Pour trouver une réponse à cette question, il faut évidemment examiner les écrits des linguistes les plus influents parmi les linguistes contemporains.

La première question que se pose le traducteur au sujet de la linguistique est: quelle place la traduction occupe-t-elle dans celle-ci, c'est-à-dire dans les préoccupations des linguistes, en particulier des linguistes les plus connus? Pas la moindre, selon Mounin. «Aucun des linguistes qui sont à l'origine des

14. Étienne Dolet (1963) : «La manière de bien traduire d'une langue en une autre », dans E. Cary, les Grands traducteurs français, Genève, Georg.

15. Delisle, op cit., p. 96 
tendances actuelles, dit-il, n'a consacré la moindre place à l'examen de cette opération... ${ }^{16}$ " Mais, pour en avoir le cœur net, examinons quand même attentivement les écrits de ces auteurs. À commencer, naturellement, par F. de Saussure, le «père» de la linguistique moderne.

A la fin du Cours de linguistique générale, Saussure nous dit que «la linguistique a pour unique et véritable objet la langue envisagée en elle-même et pour elle-même ${ }^{17} \%$. (Les mots à souligner sont unique et en elle-même et pour elle même.) Saussure ne parle de traduction nulle part. Il mentionne la «linguistique externe» et reconnaît que cette linguistique s'occupe «des choses importantes» et que «c'est surtout à elle que l'on pense quand on aborde l'étude du langage», mais il précise que de sa définition de la langue «s'écarte tout ce qu'on désigne par linguistique externe ${ }^{18} »$. Il fait une référence indirecte à la traduction dans le cadre de la discussion de la syntaxe: « une langue, dit-il, exprime quelquefois la succession des termes par une idée qu'une autre rendra par un ou plusieurs termes concrets ${ }^{19}$ ". Et c'est tout.

Parmi les autres linguistes d'expression française les plus connus, A. Martinet est l'un des très rares à parler de traduction, mais dans Éléments de linguistique générale il ne lui consacre qu'une page, et ce, seulement pour souligner «les dangers auxquels nous expose la nécessité, pour comprendre une autre langue, de traduire chaque énoncé dans la nôtre, c'est-à-dire de réarticuler l'expérience étrangère selon le modèle qui nous est familier ${ }^{20} \%$. Il faudra attendre J. Darbelnet pour voir la traduction reconnue comme une discipline distincte à laquelle on devrait accorder «une inscription normale dans le cadre de la linguistique ${ }^{21} \gg$. Il y a, évidemment, Mounin. Mais son Linguistique et traduction est surtout une exhortation des traducteurs à la «réflexion théorique» (linguistique), et des linguistes à se pencher sur les problèmes que la traduction pose à la linguistique. Du point de vue traductionologique, ce livre ne contient que des généralités. Quant à Problèmes théoriques de la traduction, Ladmiral lui portera un jugement sévère. On peut reprocher à Mounin, dit Ladmiral, de «traiter des problèmes théoriques de la traduction dans l'esprit de ce qui mériterait de s'appeler un "cours de linguistique générale» didactique sans jamais rien qui ressemble à la moindre référence à la pratique de la traduction ${ }^{22} \gg$.

Si nous passons à Louis Hjelmslev, chef de file des linguistes danois, nous trouvons également que s'il n'ignore pas totalement la traduction, ses préoccupations sont ailleurs. Dans le Langage, Hjelmslev ne dit rien qui intéresse vraiment le traducteur, et dans Prolégomènes à une théorie linguistique, il expose uniquement une méthodologie (empiriciste) en matière linguistique ${ }^{23}$,

16. Mounin, Linguistique et traduction, p. 63.

17. F. De Saussure (1975): Cours de linguistique générale, Paris, Payot, p. 371.

18. Ibid., p. 41

19. Cours, p. 191.

20. André Martinet (1970) : Éléments de linguistique générale, Paris, Armand Colin, (1960), p. 37.

21. J.P. Vinay et J. Darbelnet (1975) : Stylistique comparée de l'anglais et du français, Montréal, Beauchemin, (1958), p. 23. Ce livre a comme sous-titre: Méthode de traduction.

22. Ladmiral, op. cit., p. 174.

23. Louis Hjelmslev (1966) : le Langage, Paris, Editions de Minuit, 1966, et Prolégomènes à une théorie du langage (1971) : Paris, Editions de Minuit. 
que Firth appellera «philosophie linguistique ${ }^{24} \gg$. Dans Essais linguistiques, il fait deux références à la traduction, dont l'une est pertinente et l'autre fait plutôt sourire. Parlant de la «mise en forme de différentes manières dans les langues différentes» d'une seule et même «zone de substance», il souligne que «ces différences entre les distinctions établies dans les systèmes de la forme linguistique sont la pierre d'achoppement dans toute traduction ${ }^{25}$ ». L'autre référence, que nous ne mentionnons que parce qu'elle est amusante, fera sans doute froncer les sourcils des politologues et des sociologues. Le défaut de compréhension entre le monde communiste et le monde occidental, dit-il, est «en dernière analyse (sic!)... une question de traduction ${ }^{26} »$.

Après l'école danoise, il y a le Cercle de Prague. La réputation de ce cercle est fondée sur ses travaux sur la phonologie. Cependant, l'un de ses membres, Roman Jakobson, retient l'attention, car il est l'un des rares linguistes à s'intéresser spécialement à la traduction. Il aborde celle-ci sous trois angles différents : linguistique, anthropologie, et communication. Dans Essais de Linguistique générale, Jakobson nous offre trois études qui ont pour titres : "Le langage commun des linguistes et des anthropologues», "Aspects linguistiques de la traduction» et «Linguistique et théorie de la communication», dans lesquels il traite des problèmes de bilinguisme et de traduction ${ }^{27}$.

Avec Jakobson, qui est à la fois européen et américain, on peut passer aux linguistes américains, à commencer par Léonard Bloomfield, le chef de file des structuralistes. Pendant trois décennies (1930-1960), il a dominé la linguistique américaine. Dans son important ouvrage, Le langage, Bloomfield ne mentionne la traduction que deux fois: une fois pour dire que pour expliquer à quelqu'un qui comprend le français ce qu'est «an apple», on peut se servir de la traduction et lui dire qu'il s'agit d'une pomme ${ }^{28}:$ l'autre fois, pour expliquer qu'il est difficile de rendre le sens des «formes" étrangères parce que «les unités sémantiques de différentes langues ne concordent pas...» et que la traduction de la langue natale ne peut manquer d'égarer le débutant ${ }^{29} \gg$. Avec Zellig Harris, élève de Bloomfield, la question de la traduction ne se pose même pas parce que, pour appliquer scientifiquement les «procédures de découverte» qu'il préconise, on ne peut étudier qu'une langue, ou même un dialecte, à la fois. «L'univers du discours dans une étude linguistique descriptive, écrit-il dans Structural Linguistics, est une langue ou un dialecte seulement ${ }^{30} \%$. Un autre structuraliste américain bien connu, C.F. Hockett, ne fait que trois brèves références à la traduction dans son important ouvrage, $A$ Course in Modern Linguistics. D'autre part, s'il nous dit que l'étude de la linguistique est utile parce que celle-ci a des applications dans divers domaines, parmi les dix qu'il mentionne explicitement, la traduction ne figure pas ${ }^{31}$. Quant à l'école transformationnelle-

24. Dans R.F. Palmer (1968) : Selected Papers of J. R. Firth, 1952-1959, London, Longmans, p. 44.

25. Louis Hjelmslev (1971): Essais linguistiques, Paris, Éditions de Minuit, p. 100.

26. Ibid., p. 102.

27. Roman Jakobson (1963) : Essais de linguistique générale, Paris, Éditions de Minuit.

28. Leonard Bloomfield (1970) : le Langage, Paris, Payot, (1933), p. 133.

29. Bloomfield, op. cit., p. 477.

30. Zellig Harris, Structural Linguistics (1966) : Chicago, University of Chicago Press, Phoenix Books, (1951).

31. C. F. Hockett (1968): A Course in Modern Linguistics, New York, Macmillan, p. 1 et 2. 
générative, bien que ses recherches soient étroitement liées aux besoins de la traduction, il s'agit essentiellement de la traduction mécanique - qui est loin des préoccupations du traducteur ordinaire. En tous cas, en grammaire transformationnelle-générative, on n'étudie qu'une seule langue à la fois. La « réécriture » ne s'applique qu'à l'intérieur d'une seule langue. La traduction est exclue d'une telle grammaire. Il n'est donc pas étonnant que dans les principaux ouvrages de Noam Chomsky, Structures syntaxiques, Aspects de la théorie syntaxique, la Pensée et le langage, on ne trouve nulle référence à la traduction $^{32}$. Notons enfin que parmi les linguistes américains, deux, Edward Sapir et B.L. Whorf, ont apporté une contribution importante à l'éclaircissement de certains problèmes de traduction, mais ce sont plutôt des ethnolinguistes et ils n'ont pas parlé de traduction en tant que telle dans leurs importants ouvrages $^{33}$. Il faut naturellement mentionner Nida. Comme nous l'avons signalé plus haut, il est sans doute celui qui a contribué le plus à l'éclaircissement de beaucoup de questions, tant théoriques que pratiques, de traduction, bien qu'il se penche essentiellement sur les problèmes de traduction de la Bible, qui est un genre plutôt spécial ${ }^{34}$.

Jusqu'à présent, nous avons laissé de côté les linguistes britanniques. Il nous faut maintenant en parler. Du point de vue de la traduction, ils forment un groupe assez intéressant. Malinowski a été déjà mentionné (voir p. 130). Après lui vient Firth. Comme Jakobson, Firth s'est intéressé spécialement aux problèmes de traduction. En plus des références constantes au «contexte situationnel» dans The Tongues of Men and Speech ${ }^{35}$, il a étudié la traduction en tant que telle dans une série d'essais remarquables, dont deux portent spécifiquement sur la linguistique et la traduction: "Linguistic analysis and translation» et «Linguistics and translation ${ }^{36}$ ». Dans le sillage de Firth, M.A.K. Halliday, chef de file des "néo-firthiens", s'est penché sur la traduction, bien que davantage sous l'angle de l'enseignement des langues. Il lui a consacré un chapitre dans The Linguistic Sciences and Language Teaching, et un autre dans Patterns of Language ${ }^{37}$. Un autre "néo-firthien", J.C. Catford, qui se réclame à la fois de Firth et de Halliday, a essayé d'appliquer systématiquement la théorie linguistique à l'analyse de la traduction dans $A$ Linguistic Theory of Translation $^{38}$. A part les quatre auteurs mentionnés, les autres se sont peu inté-

32. Noam Chomsky (1969) : Structures syntaxiques, Paris, Seuil, Aspects de la théorie syntaxique (1971) : Paris, Seuil, La pensée et le langage (1968): Paris, Payot.

33. Edward Sapir (1971) : Language, London, Ruper Hart-Davis, (voir chapitre sur «Language, Race and Culture»); et Linguistique (1968) : Paris, Éditions de Minuit, (voir partie «Linguistique générale »); Benjamin Lee Whorf (1976): Language, Thought, and Reality, Cambridge, Mass., MIT Press, (1956), (voir chapitres sur «Linguistics as an Exact Science», et «Language, Mind and Reality").

34. Eugene Nida and Charles Taber (1974): The Theory and Practice of Translation, Leiden, E.J. Brill; Eugene A. Nida (1964): Toward a Science of Translating, Leiden, E.J. Brill et Componential Analysis of Meaning (1975) : Paris, Mouton.

35. Op. cit.

36. Dans R. F. Palmer, op. cit.

37. M.A.K. Halliday, Angus McIntosh, and Peter Strevens (1964): The Linguistics Sciences and Language Teaching, Bloomington, Indiana University Press; et Angus McIntosh and M.A.K. Halliday (1967) : Patterns of Language: Papers in General, Descriptive and Applied Linguistics, Bloomington, Indiana University Press.

38. J.C. Catford (1974) : A Linguistic Theory of Translation, London, Oxford University Press. 
ressés à la question. John Lyons, dans un livre important, Introduction to Theoretical Linguistics, y fait une dizaine de brèves références dont la plus intéressante est: "Toutes sortes de problèmes sémantiques intéressants sont en jeu en traduction ${ }^{39} »$. Un autre linguiste britannique éminent, R. H. Robins, dans Linguistique générale : une introduction, montre plus d'intérêt pour le sujet, mais il n'a consacré que deux pages et demie à celle-ci, dans lesquelles il s'est contenté de remarques assez générales et reconnaît, d'ailleurs, que «bien que les principes de la traduction soient du domaine de la science linguistique, sa réalisation effective relève plus de l'art... ${ }^{40}$ »

Examinons maintenant comment l'attitude des linguistes que nous venons de passer en revue se reflète sur les points particuliers qui intéressent spécifiquement le traducteur.

En ce qui concerne la forme du langage, ce n'est pas la langue écrite, mais le médium sonore, phonique, qui constitue l'objet essentiel de l'étude des linguistes contemporains; si les termes «phonèmes», «morpho-phonologie» sont employés constamment par eux, les termes «graphèmes », «morphographologie» ne le sont presque jamais. "L'objet de la linguistique, dit Saussure, n'est pas défini par la combinaison du mot écrit et du mot parlé; ce dernier constitue à lui seul cet objet ${ }^{41}$ " (Soulignons : à lui seul). Bally se fait l'écho de cette vue en disant que «la langue est faite surtout pour l'usage oral et ce serait une faute de ne pas prendre celui-ci comme norme ${ }^{42} \gg$. Quant à Martinet, s'il reconnaît que «les différences entre la graphie et la phonie sont de nature telle que l'on peut dire, sans aucune exception, que la structure écrite ne se confond pas avec celle de la langue parlée... ${ }^{43}$ » il définit cependant la langue comme «un instrument de communication doublement articulé et de caractère vocal» (soulignons vocal), et souligne que «le linguiste fait donc, par principe, abstraction des faits de graphie ${ }^{44} »$. Pour lui, les unités linguistiques sont les monèmes, qui sont des unités «douées d'un contenu sémantique et d'une expression phonique» (soulignons phonique) ${ }^{45}$.

Du côté américain, Bloomfield est très catégorique en la matière. «L'écriture, dit-il, n'est pas la langue, mais un simple moyen d'enregistrer la langue au moyen des signes visibles ${ }^{46}$ », et quand il parle de «forme linguistique», il entend par là «une forme phonétique qui a un sens» (soulignons phonétique) ${ }^{47}$. «Writing is not language» sera repris sous la forme d'un slogan qui résonnera à travers toute la linguistique américaine contemporaine. Hockett, pour sa

39. John Lyons (1977): Introduction to Theoretical Linguistics, Cambridge, Cambridge University Press, (1968), p. 409.

40. R. H. Robins : Linguistique générale : une introduction », Paris, Armand Colin.

41. Saussure, op. cit., p. 45.

42. Bally (1950): Linguistique générale et linguistique française, Paris, A. Frank (1932), p. 24.

43. Martinet, dans Jeanne Martinet, op. cit., p. 161

44. Ibid., Éléments de linguistique générale, p. 5.

45. Martinet, op. cit., p. 20.

46. Bloomfield, op. cit., p. 25.

47. Ibid., p. 131. 
part, considère que les trois principaux sujets d'étude du linguiste sont la grammaire, la phonologie et la morpho-phonémique (l'accent ici étant sur phono $)^{48}$. Cette préoccupation exclusive pour la phonologie est aussi évidente chez Chomsky, pour qui les trois composantes de la grammaire transformationnelle générative sont: la syntaxe, la phonologie et la sémantique ${ }^{49}$, et la tâche du grammairien consiste à établir des règles permettant de relier le son au sens (de nouveau, l'accent est sur phono et son).

Si nous nous tournons vers les linguistes britanniques, nous trouvons une divergence de vues très nette avec leurs confrères américains. Pour eux, la langue écrite ne peut être exclue des préoccupations des linguistes. Ainsi, pour Firth, «la priorité scientifique ne peut être donnée à la langue parlée au dépens de la langue écrite... En traitant son matériel, le linguiste doit s'occuper aussi attentivement des formes et des systèmes écrits ou d'orthographie qui existent ${ }^{50}$.» Le point de vue selon lequel la langue parlée est principale et la langue écrite une simple représentation de celle-ci est rejetée aussi avec force par Halliday, qui n'hésite pas à dire : «J'affirmerai qu'un système de langue écrite organisée a un statut équivalent à n'importe quel «vis-à-vis» parlé qu'elle puisse avoir; ce système est simplement l'expression de la langue dans une autre substance - une expression qui a plus d'attrait pour l'œil que pour l'oreille. Autrement dit , il n'est pas simplement un système de second degré... ${ }^{51}{ }$ Halliday, notonsle en passant, est l'un des rares linguistes à parler de «graphologie».

Le refus de Firth et de Halliday d'exclure la langue écrite des préoccupations du linguiste est partagé par d'autres éminents linguistes britanniques, dont Lyons et Robins. Selon Lyons, il y a des différences entre la langue écrite et la langue parlée et, par conséquent, «la langue écrite ne peut être considérée comme un simple transfert de la langue parlée à un autre medium ${ }^{52}$, et «tout commentaire sur la langue et son rôle dans la société moderne doit reconnaître que les langues écrites, bien qu'elles proviennent dans tous les cas des langues parlées, ont à l'heure actuelle un degré considérable d'indépendance structurelle et fonctionnelle ${ }^{53} \gg$. Robins, pour sa part, considère que «la parole et l'écriture sont deux modes essentiels de communication dans le champ d'investigation linguistique... ${ }^{54}$ » Pour ce genre d'étude il suggère le terme «graphonomie».

Le refus dont nous venons de parler n'est, cependant, qu'un refus de principe, qui n'a pas de suite du point de vue pratique: les ouvrages des auteurs en question contiennent toujours des études sur la phonétique, la phonologie et la morphophonologie, mais pas sur la graphologie et la morphographologie.

Passons maintenant à un domaine beaucoup plus important, celui du sens. En quoi la linguistique contemporaine peut éclairer le traducteur sur ce point? $\mathrm{Si}$ nous prenons la linguistique américaine, peu de choses avant 1965. Cette

48. Hockett, op. cit., p. 13.

49. Chomsky, Aspects de la théorie syntaxique, p. 16.

50. Firth, dans Palmer, op. cit., pp. 30-31.

51. Halliday, Patterns of Language, p. 99.

52. Lyons, op. cit., pp. 41-43.

53. Ibid. Éléments de sémantique, p. 62.

54. Robins, op. cit., p. 113. 
linguistique, dans le sillage de Bloomfield, écarte systématiquement l'étude de la signification de ses préoccupations. Cette linguistique se veut formelle, et ne s'occupe que de formes. Pour Bloomfield, si on peut définir les termes dont on possède une connaissance scientifique (comme le sel, $\mathrm{NaCl}$ ), il n'y a aucun moyen de définir les mots comme «amour», «haine» - qui forment la majorité. «Pour donner une définition exacte de la signification de chaque forme d'une langue, il nous faudrait un savoir scientifiquement exact de tout ce qui forme l'univers du locuteur, ce qui est impossible ${ }^{55} »$. Il en conclut que «les sens ne peuvent pas être définis dans les termes de notre science» et il faut laisser cette tâche aux chercheurs des autres sciences et au "savoir commun ${ }^{56}$ ". Pour Hockett, l'étude du sens, la sémantique, comme la phonétique, n'est qu'un sous-système périphérique. "La linguistique, dit-il, a toujours porté son attention principalement aux sous-systèmes centraux, sans faire trop attention aux sous-systèmes périphériques» parce que «ces derniers sont plus difficiles à étudier et que, jusqu'à présent, on sait peu de choses sur eux ${ }^{57} \gg$. Un autre structuraliste, J.B. Carroll, adopte une attitude tout aussi rigide. "Le linguiste, dit-il, refuse de s'occuper, excepté indirectement, de la teneur de la communication, c'est-à-dire de ce dont on parle»; il ne s'intéresse qu'au «véhicule de la communication...» parce que s'il s'occupe de la teneur de la communication, «il devra s'occuper de la totalité du savoir humain ${ }^{58}$ ».

La position de Bloomfield, Hockett, et Carroll, sera aussi celle de Chomsky jusqu'en 1965. En 1957, dans Structures syntaxiques, Chomsky précise que «la notion de «grammatical» ne peut être assimilée à celle de «doué de sens» ou «significatif» dans quelque sens que ce soit», que «toute recherche d'une définition de la grammaticalité fondée sur la sémantique serait vaine ${ }^{59}$ ». Il reconnaît «l'indéniable intérêt et l'importance» des études sémantiques, mais déclare qu'il se voit forcé de conclure que «la grammaire est autonome et indépendante du sens ${ }^{60} »$. Cette position sera abandonnée par lui en 1965 dans Aspects de la Théorie syntaxique. Dans la nouvelle approche, la composante sêmantique sera l'une des trois composantes "principales» de la grammaire transformationnelle-générative (les deux autres étant la phonologie et la syntaxe) et l'interprétation sémantique deviendra une préoccupation de linguiste en analyse grammaticale ${ }^{61}$. Cette réhabilitation de la sémantique donnera une forte impulsion aux études sémantiques aux États-Unis, en particulier à l'analyse componentielle, surtout avec J. Fodor et J. Katz ${ }^{62}$.

En Europe, les linguistes n'ont pas adopté une attitude de rejet systématique de la sémantique. E. Benveniste, rappelant la définition saussurienne du

55. Bloomfield, op. cit., p. 138.

56. Bloomfield, op. cit., p. 138.

57. Hockett, op. cit., p. 137.

58. J.B. Carroll (1968): The Study of Language, Cambridge, Harvard University Press, (1953), p. 12.

59. Chomsky, Structures syntaxiques, p. 17-18.

60. Ibid., p. 19

61. Ibid., Aspects de la théorie syntaxique, p. 16.

62. Jerry A. Fodor et Jerrold J. Katz (1964): The Structure of Language, Englewood Cliffs, N.J. Voir les chapitres sous «The Structure of a Semantic Theory» et «Analicity and Contradiction in Natural Language $»$. 
signe, souligne que «le langage, sous quelque point de vue qu'on l'étudie, est toujours un objet double, formé de deux parties, dont l'une ne vaut que par l'autre ${ }^{63}$ ». Martinet, pour sa part, est sceptique à l'égard de «certains linguistes quil se sont fixé comme idéal la mise au point d'une méthode de description qui ne ferait pas intervenir le sens des unités significatives ${ }^{64} \gg$. On ne saurait recommander, dit-il, «une méthode qui fait totale abstraction du sens des unités significatives ${ }^{65}$ ». Notons que c'est en France, avec Michel Bréal, qu'est née la sémantique moderne. Hors de France, cependant, l'attitude n'est pas la même. Hjelmslev partage le point de vue des structuralistes américains, et déclare que "le sens est inaccessible à la connaissance», que le linguiste n'analyse que la forme des langues et qu'il «appartient aux autres sciences d'en analyser le $\operatorname{sens}^{66} \gg$.

Outre-Manche, l'attitude des linguistes à l'égard de la sémantique est également positive. Firth s'intéresse spécialement aux problèmes de signification et rejette résolument les "procédures mécaniques» des structuralistes américains. Il considère que la linguistique souffre d'un "excès de phonèmes» et plaide en faveur d'un «retour au second front ", c'est-à-dire à l'intégration de la signification à la linguistique (le terme «second front» est emprunté à Jakobson) ${ }^{67}$. D'où aussi son intérêt pour Malinowski et son insistance sur la notion de «contexte situationnel». Les disciples de Firth ont continué dans cette ligne. Selon Halliday «le langage est une activité significative» et le but de la description linguistique est «d'élucider la signification linguistique à ses divers niveaux ${ }^{68}$ ». Pour lui, l'analyse formelle de la langue est en soi une étude de la signification. «Il est impossible, dit-il, de décrire la langue sans tenir compte de la signification" et "la dichotomie entre forme et sens est une proposition fausse ${ }^{69} \%$. Lyons, qui se penche vers la grammaire transformationnellegénérative, déplore cependant que «beaucoup des livres influents en linguistique qui sont apparus au cours des derniers trente ans prêtent peu ou pas d'attention à la sémantique ${ }^{70}$ ». Il est l'un des linguistes contemporains à accorder une attention spéciale à cette branche, et dans Introduction to Theoretical Linguistics, il explique qu'il a délibérément donné plus de place à la sémantique qu'à la phonétique et la phonologie que la plupart des autres manuels de linguistique. Ceci, sans doute, pour rétablir l'équilibre. Enfin, Robins considère que «les questions d'ordre sémantique sont en fait d'un intérêt primordial pour le linguiste» et que «une description grammaticale se doit de tenter de définir les types de fonctions sémantiques correspondant à des degrés divers à des éléments et des catégories d'ordre grammatical ${ }^{71}$ ».

Sur la sémantique, les points de vue sont donc assez partagés. Examinons, maintenant une question qui, du point de vue pratique, est encore

63. Émile Benveniste (1966) : Problèmes de linguistique générale, Paris, Gallimard, 2 vol., p. 41.

64. Martinet, op. cit., p. 34.

65. Ibid.

66. Hjelmslev, Prolégomènes, p. 98.

67. Firth, dans Palmer, op. cit., p. 160.

68. Halliday, Patterns of Language, p. 4.

69. Halliday, Patterns of Language, p. 40.

70. Lyons, op. cit., p. 40.

71. Robins, op. cit., p. 235. 
plus importante pour le traducteur, celle de l'objet de l'analyse linguistique. Hjelmslev a dit que Saussure a fait «la découverte de la langue» et «du même coup on prend conscience du fait que la linguistique de l'époque n'avait envisagé que la parole» et avait jusque-là négligé son "unique et véritable objet $^{72} »$. Langue, système, structure, linguistique structurale, linguistique structuraliste, grammaire transformationnelle-générative: si les linguistes contemporains sont en désaccord sur beaucoup de points, ils sont unanimes que l'objet de l'analyse linguistique est la langue, et non la parole. Chomsky, qui n'aime pas la dichotomie langue-parole, préfère parler de compétenceperformance. Mais quelle que soit l'école ou la terminologie adoptée, l'objet est le même.

Dans cette étude, l'analyse porte sur la phrase. Celle-ci, envisagée comme unité abstraite, est l'unité maximale de l'analyse grammaticale. Bloomfield la définit comme «une forme linguistique indépendante qui est incluse dans une linguistique plus large, en vertu d'une construction grammaticale quelconque ${ }^{73}$ ». Hockett adoptera une définition analogue, dans laquelle «constitute» est substituée à «construction ${ }^{74}$ ». Certains, comme Harris, préfèrent parler de "énoncé ", qu'il définit comme «une chaine de parole d'une personne, avant et après laquelle il y a silence de la part de cette personne ${ }^{75}$ ». Incidemment, un énoncé, selon cette définition, risque d'être interminable, si la personne qui énonce est Hitler ou Fidel Castro. Harris précise, cependant, que «l'analyse linguistique exacte ne va pas au-delà des limites de la phrase; les exigences de ses procédures ne permettent pas l'étude des relations entre une phrase et ses voisines, ou entre les parties d'une phrase et celles de ses voisines ${ }^{76}$ ". Pour Chomsky, la langue est «un ensemble de phrases». Mais la phrase de Chomsky est une phrase idéalisée. Il précise que «une grammaire générative n'est pas un modèle pour un locuteur et un interlocuteur», et que les questions qui concernent la façon dont le locuteur et l'interlocuteur dérivent une phrase appartiennent à la théorie de l'usage de la langue - la théorie de la performance ${ }^{77}$.

Si nous passons aux linguistes britanniques, nous constatons qu'il y a une divergence entre Firth d'une part, et le reste de l'autre. Pour Firth, «l'énoncé de la signification des mots, des phrases et des textes entiers est ce dont doit s'occuper la linguistique dans son ensemble» (c'est Firth qui souligne textes entiers) et «les systèmes et structures linguistiques sont liés aux systèmes et structures dans les événements, les objets et les personnes impliqués et ce que font ces personnes ${ }^{78}$ ». Mais pour Halliday, s'il existe trois niveaux distincts en science linguistique - substance, forme, et contexte - (soulignons contexte), la phrase est «la limite supérieure» (de l'analyse linguistique) ${ }^{79}$. Quant à Catford, s'il définit texte comme «une chaine de la langue, parlée ou écrite»

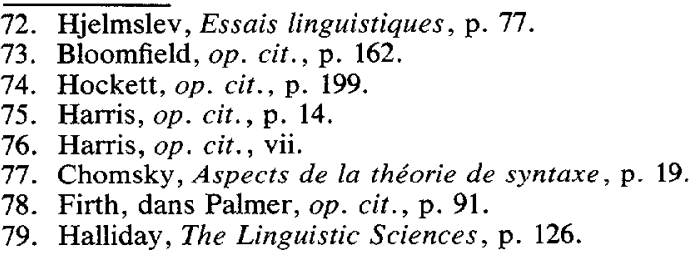


et «selon les circonstances, un texte peut être une bibliothèque entière, un seul volume, un chapitre, un paragraphe, une phrase, une proposition etc...», il adopte comme base d'analyse le «rang-échelle» (rank-scale) de Halliday, dont le niveau le plus bas est le morphème, mais dont le niveau le plus élevé est la phrase $^{80}$. Notons, enfin, que Lyons et Robins acceptent tous les deux la notion de la phrase comme unité maximale d'analyse grammaticale.

Chez les linguistes d'expression française passés en revue, la phrase est placée soit dans la parole, soit dans le discours, c'est-à-dire hors des préoccupations centrales du linguiste. Saussure se borne à dire que la phrase est «le type par excellence du syntagme», mais qu'elle appartient à la parole et non à la langue ${ }^{81}$. Bally, un des linguistes qui se penche spécialement sur la phrase, place celle-ci davantage dans la langue parlée que la langue écrite, et la définit comme "la forme la plus simple de communication d'une pensée ${ }^{82}$ ». Martinet, conséquent avec sa définition de la langue comme instrument vocal de communication, emploie le terme «énoncé » ou «signe» de préférence à phrase, et s'intéresse davantage aux plus petites qu'aux grandes unités (la double articulation en unités significatives et distinctives minima - monèmes et phonèmes -). Il reconnait que «le problème de la phrase n'a pas une place de choix parmi les préoccupations des linguistes», et ce qui explique «qu'on puisse faire de la linguistique de façon parfaitement valable sans presque parler de la phrase» est que «rien ne se trouve dans le discours qui ne soit déjà dans la phrase ${ }^{83}$ ». C'est une façon indirecte de dire que la phrase est l'unité maximale de l'analyse linguistique. Quant à Benvéniste, il considère que la phrase est «la vie même du langage en action... avec la phrase on quitte le domaine de la langue, et l'on entre dans un autre univers, celui de la langue comme instrument de communication, dont l'expression est le discours». Il place résolument la phrase dans le discours. "C'est même par là, dit-il, qu'on peut la définir: la phrase est l'unité du discours ${ }^{84}$ ».

Nous arrivons maintenant à une question qui, pour le traducteur, est un préalable dans l'étude des langues: celle de l'attitude de l'étudiant face aux langues : prescription vs description. À cet égard, Lyons exprime sans doute le point de vue unanime des linguistes contemporains quand il dit : «La première tâche du linguiste est de décrire la façon dont les gens parlent (et écrivent) en fait, non de prescrire comment ils doivent parler et écrire. Autrement dit, la linguistique (du moins en premier lieu) est descriptive, non prescriptive (normative $)^{85}$ ». La linguistique contemporaine se veut aussi scientifique, et «scientifique», dit Martinet, s'oppose à «prescriptif ${ }^{86}$ ». Scientifique veut dire aussi théorique. La description ne fait que suivre la théorie. On échaffaude d'abord une théorie, et on décrit la langue ensuite selon cette théorie, et non inversement. «Étudier la langue scientifiquement, dit Halliday, veut dire construire

80. Catford, op. cit., p. 21.

81. Saussure, Cours, p. 172.

82. Bally, op. cit., p. 35

83. A. Martinet (1974) : la Linguistique synchronique, Paris, PUF, p. 228

84. Benvéniste, op. cit., pp. 128-130.

85. Lyons, op. cit., p. 43.

86. Martinet, op. cit., p. 6. 
une théorie du mécanisme de la langue, et de là tirer certaines méthodes exactes de description des langues ${ }^{87}$ \%.

Qui dit linguistique contemporaine dit linguistique structurale. Or, comme le remarque C.G. Lipschy, la linguistique structurale est caractérisée par son abstraction et sa généralité, et «elle s'oppose à la recherche du concret et du particulier qu'une grande partie de la linguistique traditionnelle posait comme son but spécifique». Si en linguistique traditionnelle, on a souvent mis l'accent sur les actes linguistiques particuliers, aujourd'hui la linguistique préfère considérer de tels actes comme «des manifestations de la langue utilisables à titre d'exemple ${ }^{88_{»}}$. Chomsky, qui n'hésite pas à placer ses théories dans le prolongement de la grammaire traditionnelle, reproche cependant à celle-ci de négliger les régularités. Les grammaires traditionnelles, dit-il, «contiennent des listes complètes, et explicites, d'exceptions et d'irrégularités», mais quand il s'agit de processus syntaxiques réguliers et productifs, «elles ne fournissent que des exemples et des allusions ${ }^{89}$ ».

Dans le même contexte, la notion de correction est résolument rejetée par tous les linguistes contemporains. Bloomfield, qui représente la tendance extrême en la matière, considère que cette question n'est «pas fondamentale», qu'elle peut être étudiée seulement après que beaucoup d'autres choses auront été connues», et s'étonne que «les gens sans formation linguistique gaspillent beaucoup d'effort à discuter de cette question sans se mettre à l'étude du langage qui, seule, peut leur fournir des réponses ${ }^{90} \gg$. Il reproche d'autre part aux manuels de rhétorique de confondre style et «l'idée stupide de correction ${ }^{91}$ ». Hockett se contente de dire que le linguiste «n'est pas particulièrement intéressé par ce genre de questions ${ }^{92} »$. Il existe une tendance neutre, représentée par Martinet. Pour celui-ci le linguiste «se refuse aussi bien à la vertueuse indignation du puriste qu'à l'exhaltation de l'iconoclaste ${ }^{93}{ }^{\text {}}$.

La revue que nous venons de faire est certainement loin d'être exhaustive; elle nous permet cependant de tirer certaines conclusions:

1) Les linguistes contemporains, sauf de très rares exceptions, ne s'intéressent pas à la traduction;

2) L'objet d'analyse en traduction est le discours, qui est du domaine de la parole, or la linguistique contemporaine est essentiellement une linguistique de la langue;

3) Le traducteur opère sur la langue écrite, le médium graphique, or l'objet de la linguistique contemporaine est essentiellement la langue parlée, le médium phonique;

87. Halliday, The Linguistic Sciences, p. 5.

88. C. G. Lipschy (1976) : la Linguistique structurale, Paris, Payot, p. 22, 33.

89. Chomsky, Aspects de la théorie syntaxique, p. 16.

90. Bloomfield, op. cit., p, 22.

91. Ibid., p. 471 .

92. Hockett, op. cit., p. 5.

93. Martinet, op. cit., p. 7. 
4) L'unité minimale d'analyse du traducteur est la phrase, or celle-ci est l'unité maximale d'analyse en linguistique contemporaine;

5) Le sens est au centre des préoccupations du traducteur, or, jusqu'à récemment, la linguistique contemporaine a délaissé la sémantique pour s'occuper surtout de morphologie et de taxonomie;

6) Le traducteur s'intéresse essentiellement à la grammaire prescriptive (normative), or le linguiste contemporain s'intéresse essentiellement à la grammaire descriptive (théorique).

Il résulte de ce qui précède que, apparemment, le traducteur n'a rien, ou presque rien, à attendre de la linguistique contemporaine. Mais, en fait, cette conclusion n'est pas entièrement exacte, et ce, pour un certain nombre de raisons.

D'abord, tout en affirmant que l'objet de leur étude est la langue parlée, les linguistes ont exposé leurs idées sous forme écrite, et surtout donné leurs exemples sous forme écrite, graphique et non phonétique. C'est ce que Ian Robinson a souligné quand il dit qu'il ne comprend pas le dédain des linguistes pour la langue écrite puisque «même quand ils décrivent la langue parlée les exemples que donnent leurs livres sont toujours, comme par hasard, présentés sous forme écrite et non sous forme sonore.» Il ajoute que l'analyse syntaxique s'applique toujours à la langue écrite, et que la phrase appartient à la langue écrite et non à la langue parlée ${ }^{94}$. Un certain nombre de linguistes, en particulier les britanniques, reconnaissent que la langue écrite et la langue parlée sont indépendantes. Lyons se plaint d'ailleurs que, à la différence des écoles tchèque et danoise, «les post-bloomfieldiens, $y$ compris Chomsky, ont mis l'accent sur la langue écrite qui revient à dénaturer les faits ${ }^{95} \gg$. Donc, en cette matière, le traducteur ne doit pas trop prendre au sérieux les déclarations des linguistes qui affirment qu'ils n'étudient que la langue parlée.

Ensuite, en ce qui concerne le sens, Martinet note que «aucun linguiste ne semble s'être avisé d'analyser et de décrire une langue à laquelle il ne comprend rien ${ }^{96}{ }^{\prime}$. En fait, si les structuralistes américains excluent le sens de leur préoccupation, ce n'est pas le cas des linguistes européens en général, et des linguistes chomskyens après 1965. Mais même dans le cas des bloomfieldiens, le rejet du sens hors de la linguistique n'est qu'un geste doctrinaire. En effet, il y a une flagrante contradiction entre ce qu'ils prêchent et ce qu'ils pratiquent. Quand Bloomfield définit une forme linguistique comme «une forme phonétique qui a un sens» (soulignons qui a un sens), que «la phonétique pratique et la phonologie présupposent une connaissance du signifié» et que «sans cette connaissance, nous ne pouvons déterminer les traits phonémiques ${ }^{97}{ }^{\prime}$, on se demande s'il faut prendre son rejet du sens au sérieux. Quoi qu'il en soit, l'approche des bloomfieldiens est sévèrement critiquée par d'autres linguistes. Palmer, qui considère cette approche «perverse», écrit: «en rétrospective, aujourd'hui,

94. Ian Robinson (1975): The New Grammarians' Funeral: A Critique of Chomsky's Linguistics, Cambridge, Cambridge University Press, p. 174.

95. Lyons, Eléments de sémantique, p. 62.

96. Martinet, op. cit., p. 34.

97. Bloomfield, op. cit., p. 131. 
ce point de vue théorique semble presque incroyable ${ }^{98}$ ». Il est donc clair qu'une linguistique qui enlève le sens au langage lui enlève toute substance et du même coup, sa raison d'être. Une telle linguistique n'existe pas parce qu'elle ne peut pas exister. Un signifiant sans signifié est insignifiant. C'est d'ailleurs ce qui forcera Chomsky a réintroduire la signification dans sa théorie en 1965.

Il y a cependant deux questions sur lesquelles la théorie et la pratique des linguistes contemporains coïncident : celles de l'opposition langue-parole et de prescription-description. Le linguiste contemporain étudie surtout la langue, et dans cette étude il s'occupe surtout de ce qui se passe à l'intérieur de la phrase. Mais la phrase qu'il étudie est abstraite, décontextualisée, c'est-à-dire détachée de la parole. Parallèlement, les structuralistes s'intéressent davantage à la morphologie (taxonomique) qu'à la syntaxe, c'est-à-dire davantage au fractionnement qu'à la construction de la phrase, davantage aux parties qu'au tout. La syntaxe, dit un linguiste américain cité par Palmer, est «cette partie de la linguistique dont tout le monde espérait que quelqu'un d'autre s'occuperait». Toujours selon Palmer, si, apparemment ils ont réussi à établir la plus petite unité grammaticale «les linguistes ont peu de chose à contribuer à l'analyse totale de la phrase ${ }^{99} »$. Les chomskyens, qui s'intéressent spécialement à la syntaxe, le font dans le cadre de la compétence, et la phrase qu'ils veulent étudier est idéalisée, donc également abstraite, décontextualisée, détachée de la parole.

Le traducteur, par contre, s'occupe essentiellement de parole. Mais si la parole est une actualisation de la langue, elle s'actualise, à son tour, dans le discours. Or, le discours s'insère toujours dans un contexte situationnel bien défini, qui déborde largement non seulement du cadre de la phrase, mais aussi de celui du discours et même de la langue. Sans une parfaite connaissance de ce contexte, de la situation dans laquelle le discours a été conçu, il est impossible de traduire adéquatement, c'est-à-dire d'arriver à une vraie équivalence, l'équivalence fonctionnelle. La connaissance de la langue, la compétence linguistique, n'est qu'une des nombreuses connaissances exigées du traducteur. Autrement dit, la compétence linguistique n'est qu'une des nombreuses composantes de la compétence traductive.

La connaissance de la langue implique, entre autres, une solide connaissance de la grammaire. Or la grammaire qui intéresse le traducteur, linguiste praticien, est la grammaire normative (prescriptive). Il est vrai que, avant de prescrire, il faut d'abord décrire. Mais en linguistique contemporaine, non seulement diverses écoles proposent diverses méthodes de description, mais aucune d'entre elles n'a pu offrir une description complète d'aucune langue. «Aucune théorie linguistique, note David Crystal, n'a fait l'objet d'une étude vraiment complète à présent; ce qu'on a, dans la plupart des cas, n'est que sommaire et programme ${ }^{100} »$. Même pour l'anglais, la plus analysée des langues du monde, il n'existe aucune grammaire complète, et «cela est une preuve des limitations

98. Frank Palmer (1976) : Grammar, Penguin Books, (1971), p. 110.

99. Ibid., p. 124.

100. David Crystal (1976) : Linguistics, Penguin Books, p. 120. 
évidentes de l'applicabilité de la linguistique à l'heure actuelle ${ }^{101} »$. Le traducteur est donc forcé de se rabattre sur la grammaire traditionnelle, non seulement parce qu'elle est prescriptive, mais parce qu'elle existe, et répond bien à ses besoins.

Les linguistes contemporains reprochent beaucoup de choses à la grammaire traditionnelle. Crystal a dressé une longue liste des reproches qu'on fait à celle-ci ${ }^{102}$. On l'accuse d'être "notionnelle» (fondée sur le sens), de prendre la parole comme l'objet de son étude, de s'occuper essentiellement de la forme écrite de la langue et en particulier de la langue littéraire, d'être prescriptive, et, en enseignement, d'adopter la méthode «grammaire-traduction». Mais tout cela fait que la grammaire traditionnelle répond parfaitement aux besoins du traducteur.

Il résulte de ce qui précède que si l'on veut «inscrire la traduction dans la linguistique », ou inversement, il est nécessaire d'élargir le cadre de la linguistique contemporaine et lui faire embrasser la parole, la langue écrite, la sémantique et les domaines connexes - anthropologie, sociologie, psychologie -, la stylistique, les théories de communication et d'information, et d'autres choses encore. Ce serait peut-être lui demander beaucoup.

Entre-temps, un élargissement partiel a été réalisé par l'addition d'une linguistique de la parole, comme l'a fait Bally; par l'introduction de la notion de contexte de situation, comme l'ont fait Malinowski, Sapir, Whorf et Firth; enfìn, par la réhabilitation de la linguistique traditionnelle, comme l'a fait Chomsky. Ce dernier point mérite d'être souligné. Chomsky, dans le Langage et la pensée, qualifie de «totalement fausses» les accusations portées par Bloomfield et d'autres contre la grammaire philosophique quand ils la décrivent comme basée sur un modèle latin, comme prescriptive, comme ne montrant aucun intérêt pour les sons de la langue, comme tendant à confondre la langue écrite et la langue parlée, et considère «qu'il est important de dissiper ces mythes pour rendre possible une appréciation objective de ce qui a été accompli $^{103}$ ». Dans Linguistiques cartésiennes, Chomsky affirme d'ailleurs que, à de nombreux égards, il lui semble tout à fait exact de considérer la théorie de la grammaire transformationnelle-générative comme elle se développe dans les travaux actuels, comme «essentiellement une version moderne et explicite de la théorie de Port-Royal ${ }^{104}$ ».

La réhabilitation de la linguistique traditionnelle, comme les dénonciations des «perversités» de la linguistique structuraliste, libèrent le traducteur d'un grand dilemme : celui d'avoir à choisir entre deux linguistiques quand il veut aborder tout simplement l'étude de la linguistique. D'autre part, elle rend possible la «jonction» de la linguistique et de la traduction. Cette jonction, réalisée, comme on l'a vu, par certains linguistes comme Nida et Darbelnet, aidlera à convaincre le traducteur qu'il est non seulement possible, mais utile, et peut-être même nécessaire, pour lui d'entreprendre l'étude de la linguistique.

101. Ibid., p. 248 .

102. Ibid., pp. 57-65.

103. Chomsky, le Langage et la pensée, p. 30.

104. Ibid. (1969): Linguistiques cartésiennes, Paris, Seuil, p. 69. 
Cela soulève immédiatement la question: en quoi l'étude de la linguistique est-elle nécessaire ou utile au traducteur?

Si la traduction est loin d'être une opération purement linguistique, elle commence et se termine par des opérations linguistiques. Comme on l'a vu, toute opération traductionnelle comporte trois phases, dont la première et la dernière - sémasiologique et onomasiologique - sont d'ordre linguistique, plus spécialement sémantique et stylistique. Elles exigent des connaissances linguistiques, plus particulièrement grammaticales, ainsi qu'une capacité en analyse sémantique. Ici, une bonne connaissance de l'analyse componentielle est pertinente, puisqu'elle fournit une méthode permettant d'identifier et d'évaluer les composantes sémantiques d'une unité lexicale dans la LO et de les reproduire dans la LT. Nida nous fournit une illustration de la manière dont cette méthode peut être appliquée systématiquement à la traduction ${ }^{105}$. Notons que l'analyse componentielle est, en fait, une analyse rhétorique miniaturisée, appliquée à la phrase, au syntagme et au mot. En cela, il n'y a peut-être rien de nouveau pour ceux qui ont étudié les langues selon la méthode «traditionnelle». Une variante de l'analyse componentielle appelée «Predicative-Componential Analysis», est proposée par Geoffrey Leech ${ }^{106}$. En fait, cependant, la notion de «prédication» (argument, prédicat, argument) est pratiquement la même que celle d'analyse logique (agent, action, objet) en enseignement traditionnel. Notons aussi que l'analyse componentielle est entrée en linguistique via l'anthropologie.

Cependant, si la sémantique est nécessaire, elle est insuffisante. Ce qui explique pourquoi Nida fait appel non seulement à la linguistique, mais aussi aux théories de communication et de l'information, et tient compte de nombreux autres éléments non linguistiques, qui sont encore plus importants pour la compréhension totale comme pour la reproduction totale du message. L'analyse sémantique, pour utile qu'elle soit, ne peut aider le traducteur à résoudre toutes ses difficultés lexicales. Pour le faire, le traducteur doit chercher ailleurs. Nida reconnaît lui-même que «ces éléments non linguistiques du contexte sont entièrement hors de portée d'une théorie linguistique du sens. La solution d'une telle ambiguïté dépend des faits culturels, non des faits linguistiques ${ }^{107} \gg$. Ici, il faut faire intervenir les théories de Malinowski, Sapir, Whorf, et Firth sur le contexte de situation, sur la relation entre : langage et réalité, langage et pensée, langage et vision du monde, langage et culture. Mais avec cela, on sort du domaine de la linguistique pour entrer dans ceux de l'anthropologie, de la sociologie, de la psychologie et de la philosophie.

Nida fait aussi largement appel à la grammaire générative. La distinction structure profonde - structure superficielle est sans doute utile dans l'éclaircissement des ambiguités d'ordre structurel (grammatical) et des problèmes liés à la nominalisation et la verbalisation. À cet égard, la linguistique chomskyenne est pertinente à la traduction. Notons, cependant, que cette distinction n'est

105. Voir Toward a Science of Translating, The Theory and Practice of Translation, et Componential Analysis of Meaning.

106. Geoffrey Leech (1976) : Semantics, Penguin Books.

107. Nida, Toward a Science of Translating, p. 117. 
pas très différente de la distinction entre «sens apparent»/«sens réel», «sujet apparent»/«sujet réel», «sujet grammatical»/«sujet logique», en analyse grammaticale traditionnelle. Qu'il me soit permis d'ouvrir une parenthèse sentimentale ici pour dire qu'en écrivant ces lignes je ne puis m'empêcher de penser au professeur de français qui m'a fait faire je ne sais combien d'analyses de ce genre en classe de sixième (de l'enseignement secondaire français).

Une autre application de la linguistique à la traduction, sous l'angle stylistique, cette fois, est fournie par Vinay et Darbelnet. L'ouvrage Stylistique comparée du français et de l'anglais est, sans doute, une contribution importante à l'étude de la traduction. Cette contribution est notable surtout dans l'identification, la description et la classification des procédés de traduction, et la dotation de la traductionologie d'une terminologie appropriée. Cependant, quand Vinay et Darbelnet ramènent la traduction «à un cas particulier, à une application pratique de la stylistique comparée ${ }^{108}$ ", ils en restreignent énormément le domaine. Comme le souligne si justement Delisle, «traduire n'est pas comparer ${ }^{109}$ "; il y a une différence fondamentale entre «traduction de langue " et «traduction de texte», la première étant «un exercice comparatif» et la seconde «un exercice interprétatif ${ }^{110} »$; et l'analyse de la langue que pratiquent les stylisticiens comparatistes «reste en deçà de l'analyse du discours sur lequel se fonde toute vraie traduction ${ }^{111}$, car cette analyse est «une analyse linguistique pratiquée a posteriori ${ }^{112}$ ». D'autre part, le découpage de la phrase en «unités de traduction» ne s'applique que dans la phase sémasiologique. (I1 faudrait peut-être parler «d'unités sémantiques ».) C'est une opération que ceux qui ont une maîtrise suffisante de la langue font automatiquement et mentalement. (En fait, c'est ce qu'on apprend déjà aux enfants en lecture dans les classes élémentaires.) Mais dans la phase onomasiologique, on ne peut parler «d'unités de traduction» plus petites que la phrase, parce que c'est la reformulation de la pensée exprimée par la phrase entière, et non un alignement de fragments de phrase traduits séparément, qu'on doit réaliser. Dans cette phase, on ne fait pas de la traduction, on fait de la rédaction.

Notons enfin que le traducteur trouvera des informations intéressantes dans les ouvrages de synthèse comme Introduction to Theoretical Linguistics de Lyons, ou des études de problèmes linguistiques spécifiques, comme dans Problèmes de linguistique générale de Benveniste, mais ce genre d'ouvrages est plutôt rare. Il y a évidemment aussi les manuels de linguistique appliquée, mais jusqu'à présent, linguistique appliquée veut dire essentiellement linguistique appliquée à l'enseignement des langues. On peut citer, à titre d'exemples, Introducing Applied Linguistics de S. Pit Corder ${ }^{113}$, Initiation à la linguistique appliquée de Guy Rondeau ${ }^{114}$, Linguistique et enseignement des langues étran-

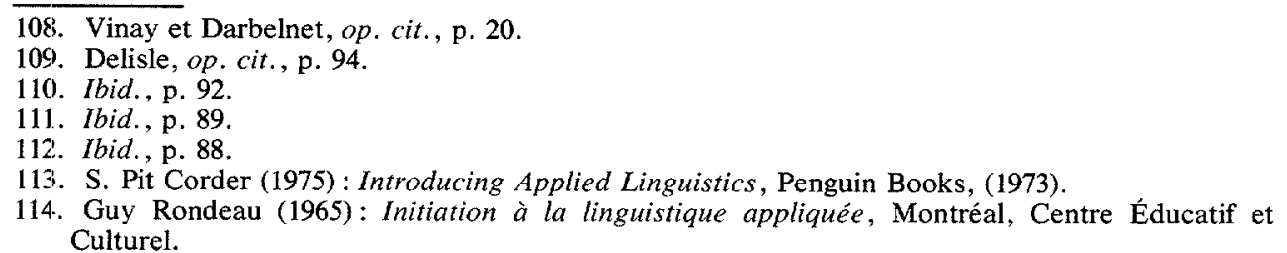


gères de $\mathrm{M}$. de Grève et $\mathrm{F}$. Van Passel ${ }^{115}$. Ce sont des ouvrages utiles certes (surtout du point de vue de la familiarisation avec la terminologie linguistique) mais qui ne touchent le traducteur que très indirectement. La linguistique appliquée est un champ encore nouveau. En fait, certains linguistes comme Palmer, disent même qu'ils «ne savent vraiment pas ce que c'est ${ }^{116}$ », et d'autres, comme Enrico Arcani, confondent critique des théories linguistiques et linguistique appliquée ${ }^{117}$. De toute façon, linguistique appliquée suppose connaissance préalable, approfondie, et simultanée de la linguistique et de la traduction; l'application de la linguistique à la traduction est donc une tâche qui ne peut être entreprise avec succès que par un linguiste doublé d'un traducteur, ou inversement - une combinaison plutôt rare à l'heure actuelle.

En attendant, il faut quand même traduire, et pour cela, le traducteur trouvera dans la linguistique traditionnelle tout ce dont il a besoin linguistiquement, quelqu'inadéquate que cette linguistique puisse être aux yeux des linguistes contemporains du point de vue de l'explication théorique. Cette linguistique est proche du traducteur parce qu'elle est «notionnelle» : fondamentalement, elle met l'accent sur la pensée et la communication. Comme le soulignent Claude Lancelot et Antoine Arnault, «les hommes ont fait des signes pour signifier leurs pensées» et c'est pourquoi «on ne peut bien comprendre les diverses sortes de signification, qui sont enfermées dans les mots, qu'on ait compris auparavant ce qui se passe dans nos pensées, puisque les mots n'ont été inventés que pour les faire connaître ${ }^{118} »$. Ce n'est peut-être pas pure coïncidence que cette pensée exprimée en 1660 , rejoint celle des traducteurs d'aujourd'hui, exprimée par Delisle: «pour expliquer l'opération traduisante dans sa totalité, le linguiste se doit d'aborder l'étude du discours et de ses rapports avec la pensée et les faits de communication ${ }^{119} »$. En matière de traduction, comme en matière de langue, chassez la pensée, et elle revient au galop.

Tout bien compté, donc, la moisson est plutôt maigre du côté de la linguistique contemporaine. D'autre part, si cette linguistique a des choses utiles à offrir au traducteur, et que ce dernier est tenté d'en entreprendre l'étude, les facteurs dissuasifs sont nombreux. Tout d'abord, le traducteur est confronté par une masse de littérature non seulement énorme, mais qui s'accroît sans cesse et rapidement. «L'accroissement quantitatif de la production linguistique est tel qu'un gros volume de bibliographie annuelle ne suffit pas à le recenser", note Benveniste ${ }^{120}$. Rien que dans le domaine de la grammaire transformationnellegénérative, dit Chomsky, (en 1970) on a assisté en cinq ans à la prolifération d'articles et de thèses «tandis qu'un flot ininterrompu de littérature parallèle fait bourdonner les ronéos». Ces travaux ont paru adopter des points de vue

115. M. de Grève et F. Van Passel (1973): Linguistique et enseignement des langues étrangères, Paris, F. Nathan.

116. Noel Minnis, edit. (1973): Linguistics at Large, St Albans, Hertfordshire, Paladin, (1971), p. 261.

117. Enrico Arcani (1972) : Principes de linguistique appliquée, Paris, Payot.

118. Claude Lancelot et Antoine Arnault (1968): Grammaire générale et raisonnée 1660, reproduction de The Scholar Press, Menston England, p. 27.

119. Delisle, op. cit., p. 70.

120. Benveniste, op. cit., p. 3. 
extrêmement divers, mais la plupart des controverses terminologiques «recouvrent, quand elles ne les enfouissent pas, des questions intéressantes ${ }^{121} »$.

Cette prolifération terminologique est un autre facteur dissuasif. "Autant de linguistes, autant de définitions", dit Bally ${ }^{122}$. En matière de terminologie, les linguistes contemporains sont mûs par un individualisme farouche. Il est évidemment tout à fait légitime de vouloir définir avec précision les termes qu'on emploie. Mais on a l'impression que, souvent, la définition devient une fin en soi, une manie qui trahit une sorte de terminologistite, dont $\mathbf{R}$. Longacre nous fournit un exemple typique ${ }^{123}$. L'accroissement quantitatif ne s'accompagne pas toujours d'un accroissement qualitatif. Faute de substance, on se débat dans les mots et sur les mots. Notons, en passant, que ceux qui se sont contentés d'une terminologie simple, et font preuve de beaucoup de modestie, comme Saussure et Bréal, sont aussi ceux qui ont eu une influence non seulement profonde mais durable. Le manque d'uniformisation en matière de terminologie est d'ailleurs, comme le souligne Crystal, «un signe évident de manque de maturité de la linguistique ${ }^{124} »$.

La profusion de terminologies n'est qu'un symptôme de la profusion de théories. "Il y a autant de linguistiques qu'il y a de linguistes», dit Hjelmslev ${ }^{125}$. Dans un ouvrage conçu pour prouver qu'en matière d'objectifs, de principes, de méthodes et de théories, malgré les différences entre divers linguistes, «il y a plus de choses sur lesquelles ceux-ci s'entendent que sur lesquelles ils ne s'entendent pas», l'auteur, J.D. Allerton, nous donne en fait un exposé qui prouve plutôt le contraire ${ }^{126}$.

Rien que la maîtrise de la terminologie consomme déjà un temps substantiel du traducteur. Mais quand celui-ci aborde la substance elle-même, il se rend vite compte qu'une bonne partie de la linguistique contemporaine n'a presque aucune pertinence pour la traduction. La phonétique, la phonologie et la morphophonologie - qui constituent une part substantielle de cette linguistique - touchent la traduction à peine. En grammaire, l'IC, la fameuse analyse en constituants immédiats (Immediate Constituent Analysis) par exemple, considérée comme une contribution majeure de Bloomfield à la linguistique, n'est, aux yeux de plusieurs autres linguistes, qu'une variante de l'analyse grammaticale traditionnelle. Elle est plus «puissante» théoriquement, mais elle suppose une connaissance préalable du sens de la phrase à découper, et elle utilise, implicitement, la catégorisation grammaticale traditionnelle, que les bloomfieldiens eux-mêmes dénoncent avec véhémence. Une autre grande découverte des structuralistes est le morphème. Mais, comme le remarque Halliday, «personne ne s'avise jamais de prendre le morphème comme unité de traduc-

121. Chomsky (1970): Questions de sémantique, Paris, Seuil, p. 33.

122. Bally, Linguistique générale, p. 29.

123. Robert E. Longacre (1968): Grammar Discovery Procedures, The Hague, Mouton, (1964) Ses définitions du syntagme, de la proposition, de la phrase sont circulaires, et pur verbiage.

124. Crystal, Linguistics, op. cit., p. 80.

125. Crystal, Linguistics, op. cit., p. 80.

125. Hjelmslev, Essais linguistiques, p. 149.

126. J.D. Allerton (1979) : Essentials of Grammatical Theory : A Consensus View of Syntax and Morphology, London, Routhledge and Kegan Paul. 
tion ${ }^{127}$ ». Quant à la grammaire transformationnelle-générative, acclamée comme une révolution en linguistique, elle est encore au stade exploratoire; elle est d'ailleurs ignorée par certains linguistes respectés comme Firth et Martinet, et, de plus, certains autres, comme Robinson, prononcent déjà ses funérailles (le titre du livre de Robinson est, rappelons-le, The New Grammarians' Funeral : A Critique of Chomsky's Linguistics). L'œuvre de Chomsky, dit Robinson, ne sera «qu'un autre épisode dans l'histoire des longs et désespérés efforts déployés pour réduire la pensée concernant le langage à une science exacte ${ }^{128} \gg$. Tout cela, inévitablement, laisse le traducteur perplexe.

Jusqu'ici, nous n'avons parlé que de la contribution de la linguistique à la traduction. Il serait peut-être approprié, en guise de conclusion, de dire quelques mots de la contribution inverse. À cet égard, ce qui frappe quand on parcourt les ouvrages de linguistique est la reconnaissance générale de la nécessité de faire appel au contexte pour déterminer le sens exact et total des unités lexicales, et ce dans une science qui accepte comme postulat que «la langue est forme et non substance». Cette notion de contexte a été introduite en linguistique, comme nous l'avons souligné, via l'anthropologie, la sémantique, et la traduction. «Dans cette perspective, remarque Bouton, on pourrait conclure sans risque d'être paradoxal, que la traduction a apporté plus à la théorie linguistique que la théorie linguistique n'a apporté à la traduction» et «lorsque le linguiste se penche sur cette opération même, il découvre qu'elle lui en apprend plus sur l'acte du langage, que la théorie dont il se réclame ${ }^{129} »$. Cette observation peut être vraie ou non, mais elle mérite d'être méditée par le traducteur face au «terrorisme linguisticiste et théoriciste » qui a dominé la linguistique ${ }^{130}$, et qui risque d'envahir aussi la traduction.

\footnotetext{
127. Halliday, The Sciences of Language, p. 128. Notons aussi que les termes "phonème», «morphème» étaient déjà utilisés par Von Humboldt en 1836, si la traduction de son livre est correcte. Voir Wilhelm Von Humboldt (1971): Linguistic Variability and Intellectual Development (traduction de George C. Buck et Frithjof A. Raven), Coral Gables, Fla., University of Miami Press.

128. Robinson, op. cit., p. 186.

129. Bouton, op. cit., p. 69.

130. Ladmiral, op. cit., p. 161.
} 